\title{
Postural adjustments and reaching in 4- and 6-month-old infants: an EMG and kinematical study
}

\author{
Victorine B. de Graaf-Peters · Hanneke Bakker • \\ Leo A. van Eykern · Bert Otten • Mijna Hadders-Algra
}

Received: 16 October 2006 / Accepted: 13 April 2007 / Published online: 16 May 2007

(C) Springer-Verlag 2007

\begin{abstract}
Adequate postural control is a prerequisite for daily activities such as reaching for an object. However, knowledge on the relationship between postural adjustments and the quality of reaching movements during human ontogeny is scarce. Therefore we evaluated the development of the relationship between the kinematic features of reaching movements and the accompanying postural adjustments in young infants. Twelve typically developing (TD) infants were assessed twice, i.e. at 4 and 6 months of age, in supine and supported sitting position. Reaching was elicited by presenting toys in the midline at an arm-length distance while simultaneously surface EMGactivity was recorded from multiple arm-, neck-, trunk- and leg muscles. Concurrently kinematics of reaching were recorded with an ELITE system; kinematic analysis was restricted to the behaviour of so-called movement units, which are sub movements of reaching determined with the help of peaks in the velocity profile of the hand, maximum movement velocity and movement duration. A computeralgorithm determined significant phasic muscle activity. Activity in neck and trunk muscles (postural activity) was related to the onset of the prime mover, which was the arm
\end{abstract}

\footnotetext{
V. B. de Graaf-Peters · H. Bakker · L. A. van Eykern ·

M. Hadders-Algra $(\square)$

Department of Neurology - Developmental Neurology, University Medical Center Groningen,

University of Groningen, Hanzeplein 1,

9713 GZ Groningen, The Netherlands

e-mail: m.hadders-algra@med.umcg.nl

B. Otten

The Center for Human Movement Sciences Groningen,

University Medical Center Groningen,

University of Groningen, Groningen,

The Netherlands
}

muscle being activated first. The results indicated that about $50 \%$ of reaching movements in lying and sitting infants aged 4 and 6 months were accompanied by direction-specific postural adjustments. At 4 months variation dominated, but at 6 months a preference to recruit muscles in a top-down order (during sitting) and in the configuration of the complete pattern, i.e. the pattern in which all dorsal neck- and trunk muscles are activated in concert, (both conditions) emerged. Interestingly, the postural characteristics such as the presence of direction-specificity, recruitment of the complete pattern and top-down recruitment, were related to how successful the reaching was and the kinematics of reaching. It was concluded that the presence of direction-specific activity is not a prerequisite for the emergence of reaching movements. Nevertheless, already from 4 months onwards a better postural control is associated with a larger success and a better quality of reaching.

Keywords Postural adjustments $\cdot$ Reaching ·

Kinematics $\cdot$ EMG $\cdot$ Infants

\section{Introduction}

Adequate postural control is a prerequisite for daily activities such as reaching. The tight link between posture and reaching is reflected by the presence of postural adjustments accompanying reaching movements (Massion 1998).

It is well known that also during infancy posture affects reaching behaviour. For instance, it has been demonstrated that young infants, who lack adequate head and trunk control, can perform aimed arm movements towards a toy when they are firmly supported in a reclined or upright sitting position (Amiel Tison and Grenier 1983; von Hofsten 1982). Without such support these aimed movements 
cannot be performed. Other studies indicated that a comparable relationship between postural support and the ability to produce successful reaching movements is present in older infants (Rochat 1992; Savelsberg and Van der Kamp 1994; Hopkins and Rönnqvist 2002).

Little is however known on the relationship between the organization of postural control and the quality of reaching during early infancy. The development of postural control and that of reaching has been studied mainly as isolated phenomena.

Kinematical characteristics of reaching have been studied in infants sitting in an infant chair or while lying supine from 3 months onwards. Initially, around 3 months of age reaching movements are characterised by variation, i.e. by irregular and fragmented trajectories, but during the following 2 months reaching trajectories rapidly become more smooth and fluent. Reaching movements also becomes more successful, i.e. they usually result in grasping of an object between 4 and 5 months of age (Van der Fits et al. $1999 \mathrm{a}, \mathrm{b})$. The major qualitative changes of reaching movements consist of an increase in movement velocity and a decrease in the number of trajectory corrections (Von Hofsten 1991; Thelen et al. 1993). These corrections are termed movement units (MU) and are submovements of reaching, which are determined with the help of peaks in the velocity profile of the hand (Von Hofsten 1991). During the first half year of life, the kinematical characteristics of reaching seem to depend on the child's position: reaching movements in supine (Fallang et al. 2000) consist of more MU than those performed in a secured sitting position (Out et al. 1997).

The development of postural control is characterised by a protracted course (Hadders-Algra 2005). Recent data of Hedberg et al. (2004) suggested that the basic level of control in the organization of postural adjustments might have an innate origin. This level of control is involved in the generation of basic direction-specific adjustments. Direction-specificity means that perturbations inducing a forward sway of the body, such as reaching movements, are accompanied by postural activity in the muscles on the dorsal side of the body, whereas perturbations inducing a backward body sway are accompanied by activity in the 'ventral' muscles (Forssberg and Hirschfeld 1994). Functional activity at the second level of control, which is involved in the fine-tuning of the basic postural pattern on the basis of multi-sensorial afferent input from somatosensory, visual, and vestibular systems, seems to emerge around 6 months of age. This modulation can be achieved in various ways, for instance, by changing the number of direction-specific muscles recruited or by modifying the order in which the direction-specific muscles are recruited (e.g. in a caudal-tocranial sequence or in a reverse order).

The studies of Van der Fits (1999a, b) indicated that postural adjustments during reaching are direction-specific from the moment the infant is able to produce reaching movements, which end in successful grasping. The direction-specific adjustments during reaching at early age are characterised by variability, for instance, by variation in which muscle is recruited and variation in recruitment order. However the Van der Fits' studies indicated that within the variation some age-specific differences in postural activity might be observed. The studies suggested that between 4 and 6 months the number of direction-specific muscles recruited decreased and after the age of 6 months it increased again. From early reaching age onwards, recruitment of postural muscles during supported sitting seemed to follow a cranio-caudal order, an organization, which became more prominent with increasing age. However, during unsupported sitting, an ability, which in general emerges between 6 and 8 months (Piper and Darah 1994), infants initially recruited postural muscles in a caudo-cranial order. This dominance of bottom-up recruitment during independent sitting disappears around the age of 2 years (Van der Heide et al. 2003). The difference in recruitment order between the two sitting conditions is an illustration of the finding that from 6 months onwards infants gradually develop the capacity to fine-tune postural activity to task constraints (Hadders-Algra 2005).

Early interdependence of control of reaching and posture is supported by a longitudinal study of four infants by Thelen and Spencer (1998). They reported an increased muscle activity in the deltoid and trapezius muscles concurrent with the onset of successful reaching. This might be interpreted as the emerging stabilization of head and shoulder providing a supporting frame for reaching. Fallang et al. (2000) who studied kinematics of reaching in supine with accompanying kinetics of postural control reported that at the age of 4 months, a better general postural stability was associated with a better reaching performance. In addition they found that at the age of 6 months the kinematic features of the reaching movements were only weakly related to the way posture in supine was organised. The authors suggested that the age-related change in the association between postural activity and reaching performance might point to the emergence of a more subtle organization of postural adjustments. Currently, no information is available on the relationship between neck-, trunk- and leg-muscle activities and the kinematics of reaching during infant development.

The aim of the present study is to evaluate the development of this relationship in supine and sitting condition at 4 and 6 months. The present study differed in three important aspects from the Van der Fits' studies. First, the present study combined EMG recording of postural muscles with the kinematics of reaching. Second, we did not record activity of one upper extremity muscle (i.e. the deltoid), but of multiple arm- and shoulder muscles, each of which could 
act as a so-called prime mover, i.e. the arm muscle recruited first during reaching. Recent data indicated that children show considerable variation in prime mover activity and that in less than half of the reaching movements the deltoid muscle is used as prime mover (Van der Heide et al. 2003). Third, we used a more precise definition of direction-specific trials. In the Van der Fits' studies a trial was classified as direction-specific when direction-specificity was present at one of the body-levels recorded, whether or not postural activity at other levels of the body was direction-specific. In the present study we used a more stringent definition of direction-specificity: postural activity at all levels of the body had to fulfil the criteria for direction specificity (see Sect. "Methods"). Recently, the more stringent definition has also been used in other studies (Van der Heide et al. 2003; Hedberg et al. 2004).

Our study addressed the following questions: (1) Do differences in study design between the Van der Fits' studies and the current study result in different findings on postural adjustments? For instance, we expected that a stricter definition of direction-specificity would reduce the number of trials fulfilling the criterion for direction-specificity. (2) Are indicators of better postural organization at early age associated with better organised reaching movements? We regarded the presence of direction specificity or a cranio-caudal recruitment for instance as indicators of better postural organization. Better reaching movements were movements consisting of fewer MUs, in which the first MU, i.e. the transport MU, covered a larger part of the movement, and movements with a higher velocity.

\section{Methods}

\section{Subjects}

Thirteen full-term healthy infants (seven girls, six boys) participated in the study. They were assessed twice, i.e. at the ages of 4 and 6 months. The children were recruited from amongst acquaintances of the investigators. The infants' gestational age at birth varied from 38 to 42 weeks postmenstrual age (median value: 39 weeks); birth weight from 2,930 to 4,280 grams (mean: 3,582 g; SD: $468 \mathrm{~g}$ ). All children had a typical motor development. The parents of the infants gave informed consent and the procedures were approved by the ethics committee of the UMCG.

\section{Protocol}

The infants were tested in two positions: lying supine and sitting upright in an infant chair. The infant chair had a back-support and a horizontal bar at the front which could be adjusted so that the bar was located at the level between midway the nipple line and umbilicus (Fig. 1). Reaching was elicited by presenting small, attractive toys in the midline and at an arm length distance. Toys were only presented when the infant was in a calm and alert behavioural state. We aimed at recording at least ten reaching movements with the right arm in each position, but when the infant became fussy or tired the session was shortened. In order to confirm neurological integrity a standardised neurological examination according to Prechtl $(1977)^{18}$ with age-specific adaptations of the norms according to Touwen $(1976)^{13}$ was carried out after each reaching session.

\section{EMG and kinematical recordings}

EMG was measured continuously during the testing session with bipolar surface electrodes with an interelectrode distance of $14 \mathrm{~mm}$ on the following muscles: deltoid (DE), pectoralis major (PM), biceps brachii (BB), triceps brachii (TB), neck flexor (NF, sternocleidomastoid), neck extensor (NE), rectus abdominis (RA), thoracal extensor (TE), lumbar extensor (LE), rectus femoris (RF), and hamstrings (HAM) on the right side of the body. DE, PM, BB and TB are referred to as arm muscles, NF, NE, RA, TE, LE, RF and HAM as postural muscles. EMG signal were acquired by means of an electro-physiological front-end amplifier (Twente Medical Systems International, Enschede, the Netherlands) The EMG activity was pre-processed and recorded continuously with POLY, a software program for long-lasting polygraphic recordings (Inspector Research
Fig. 1 Infant of 6 months in the two different positions. Note the horizontal bar of the infant chair preventing the child to fall out of the chair. Figure published with permission of the infant's caregivers
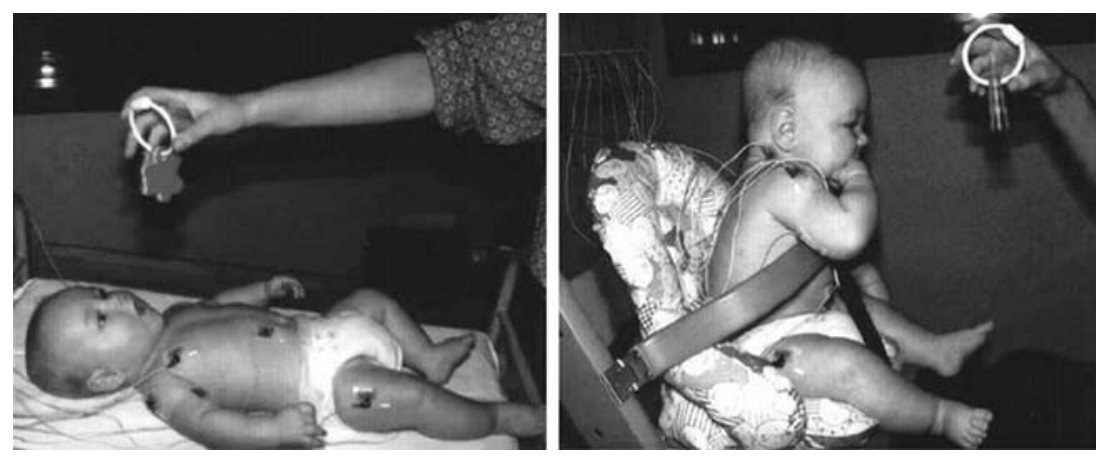
Systems, Amsterdam, the Netherlands) at a sample rate of $500 \mathrm{~Hz}$. Simultaneously, split-screen video recordings were made from a lateral and frontal view of the infant. The video registrations were time-coupled to the EMG recordings. The reaching session took about $30 \mathrm{~min}$. The numbers of trials with appropriate EMG recording included in the analyses are displayed in Table 1.

Simultaneous with the EMG recordings, movements were recorded kinematically with an ELITE system (BTS, Milan, Italy) in a two-camera configuration at a sampling frequency of $50 \mathrm{~Hz}$. A reflective marker was placed at the right side of the body on the styloid process of the radius. Sampling of the kinematical data started some seconds before toy presentation and lasted for $10 \mathrm{~s}$. The periods of kinematical sampling were indicated on the EMG recording. The numbers of trials with appropriate kinematical recording included in the analyses are displayed in Table 1.

\section{Video and EMG analysis}

The video recordings served two purposes. First, the video was used to select movements in an appropriate attentional state, performed with the right arm. Second, the video was used for classification of the behaviour of the movements of the right arm during toy presentation. Movements could be classified as pre-reaching movements (Trevarthen 1984), reaching movements which did not end in toy contact, reaching movements which did end in toy contact and reaching movements which ended in grasping of the toy. EMG and kinematical analyses were restricted to trials during which the child's reaching movement either ended in toy contact or grasping (successful reaches).

For the EMG analysis, a computer algorithm was used for the detection of phasic muscle activity. The algorithm used a derivative of the root mean square of a full-rectified signal (200 ms moving window), and marked significant deviations from a fixed detection level. The detection level was based upon a significant increase in muscle activity of a long-term (3.7 s) mean baseline activity. EMG bursts were detected when the activity exceeded the detection level for at least $50 \mathrm{~ms}$ (Van der Fits et al. 1998; 1999a, b). The activity of the neck, trunk and leg muscles was considered to be related to the arm movement when increased muscle activity was found within a time window of $200 \mathrm{~ms}$ before activation of the prime mover, i.e. the arm muscle that was activated first (virtually always DE, PM or BB) and $500 \mathrm{~ms}$ after activation of the prime mover had ended.

For each infant, each condition and each age the following parameters were calculated: (1) Percentage of directionspecific trials; direction-specificity meant that both at neck- and trunk level the 'direction-specific' (i.e., dorsal) muscle was recruited prior to the ventral muscle or without activation of the antagonistic ventral muscle. The other EMG-parameters were only calculated for trials with direction-specific postural activity. Additional EMG-parameters were: (2) Patterns of postural adjustments where patterns consist of the specific combinations in which directionspecific muscles are activated in concert. (3) The preference pattern defined as the pattern present in at least $50 \%$ of the trials. (4) The latencies of recruitment of postural muscles, defined as the time interval between the onset of the prime mover and the onset of activity in the postural muscle. For each infant, age, and position median latency values were calculated. (5) The percentage of trials with top-down recruitment. Recruitment order could only be determined when at least two direction-specific muscles showed significant phasic activity.

\section{Kinematical analysis}

Offline kinematical analysis was carried out with the help of the software package MatLab (The Mathworks Inc.) files (E. Otten, The Center for Human Movement Sciences Groningen, University of Groningen). Arm movement onset was defined as the moment at which the $3 \mathrm{D}$ velocity of the wrist increased $\geq 5 \%$ of peak velocity, whereas the moment at which $3 \mathrm{D}$ wrist velocity decreased to $\leq 5 \%$ of peak velocity was considered as the end of the movement. The data were filtered using a low-pass filter of $6 \mathrm{~Hz}$ with zero time lag. In the kinematical analysis, only trials which met the above-mentioned criteria were included; they were matched to the corresponding EMG-trial (Table 1). We used the following parameters to describe the reaching movements: (1) The number of MU per trial. A MU consisted of one acceleration and deceleration in the velocity profile of the wrist marker. (2) The duration of the

Table 1 Number of trials analysed per individual for EMG and kinematical recordings

\begin{tabular}{|c|c|c|c|c|c|c|c|c|c|c|c|c|}
\hline \multirow[t]{3}{*}{ Age } & \multicolumn{6}{|c|}{ EMG recordings } & \multicolumn{6}{|c|}{ Kinematical recordings } \\
\hline & \multicolumn{3}{|c|}{ Supine } & \multicolumn{3}{|c|}{ Sitting } & \multicolumn{3}{|c|}{ Supine } & \multicolumn{3}{|c|}{ Sitting } \\
\hline & $n$ & med & Range & $n$ & med & Range & $n$ & med & Range & $n$ & med & Range \\
\hline 4 months & 13 & 11 & $3-20$ & 12 & 9 & $4-13$ & 9 & 4 & $3-9$ & 12 & 5 & $3-10$ \\
\hline 6 months & 12 & 13 & $10-15$ & 12 & 12 & $7-17$ & 10 & 5 & $3-12$ & 12 & 6 & $4-11$ \\
\hline
\end{tabular}

$n$ number of infants, med median value 
reaching movement. (3) The relative duration of the first MU (the transport MU) in relation to total duration and (4) maximum reaching velocity.

From the kinematical parameters median values were calculated for each infant, each condition, and each age separately for trials with direction-specific postural activity and for those without. Next, for direction-specific trials median values were calculated for each infant, age and condition (1) for trials in which the pattern where all three dorsal neck and trunk postural muscles were recruited was present and for trials with another postural pattern and (2) for trials in which postural muscles were top-down recruited and for those without top-down recruitment.

\section{Statistics}

Statistical analyses were performed using the computer package SPSS (version 12.1). Due to the non-normal distribution of the data- a finding, which is typical for infancynon-parametric statistics were used. For the analyses of the effect of age and condition on the EMG and kinematical parameters the paired Wilcoxon test was used. Due to dataloss in the kinematical recordings-a well-known problem in infant research (Van der Fits et al. 1999b) -it was not possible to use the Wilcoxon test for the analyses of the kinematical data and the analyses of the relationships between the postural and reaching data. In these cases we decided to use the Mann-Whitney $U$-test. Throughout the analyses, differences with a $p<0.05$ were considered statistically significant.

\section{Results}

Postural adjustments

Figure 2 shows representative examples of postural activity during reaching at both ages in both conditions. In accordance with the Van der Fits' studies (1999a,b) we found that leg muscle activity in infants lying supine and sitting without foot support was not related to postural control. Therefore leg muscle activity was not included in the analyses. But in contrast to the previous data of Van der Fits et al. (1999a, b), who reported consistent direction-specific postural activity during reaching from 4 months onwards, we found that typically developing infants at 4 and 6 months showed direction-specific postural activity in about $50 \%$ of the trials both in supine and in the supported sitting condition. The difference in findings can be explained by the difference in definition of a direction-specific trial. The occurrence of direction-specificity was independent of the child's age and position (Table 2).

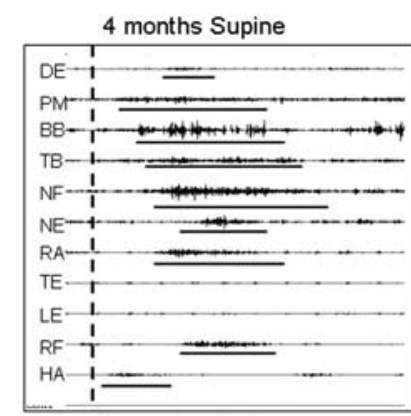

\section{6 months Supine}

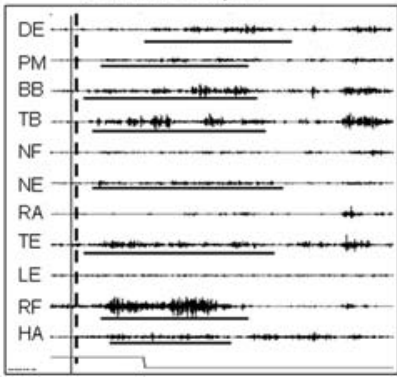

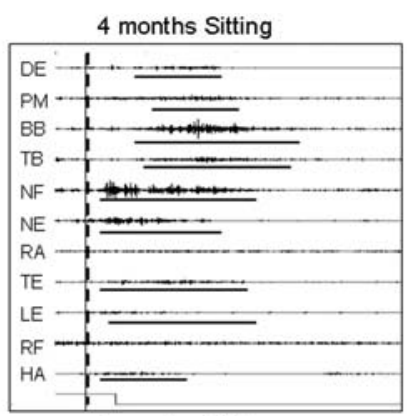

6 months Sitting

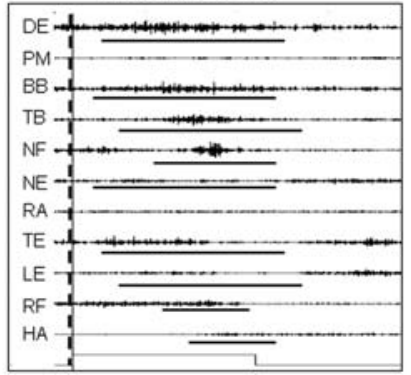

Fig. 2 Typical examples of postural EMG activity during a reaching movement in the supine and supported sitting position of an infant at 4 and 6 months of age. Each trial lasts 2,500 ms. $D E=$ Deltoid, $P M=$ Pectoralis Major, $B B=$ Biceps brachii, $T B=$ Triceps brachii, $N F=$ neck flexor, $N E=$ neck extensor, $R A=$ rectus abdominis, $T E=$ thoracal extensor, $L E=$ lumbar extensor, $R F=$ rectus femoris, $H A M=$ hamstrings. Dotted vertical lines denote the onset of the reaching movement as indicated by the kinematics. Horizontal lines delineate the presence of significant EMG bursts as defined by the computer algorithm. The prime mover is the arm muscle which first shows phasic activity, for instance the prime mover in the left hand upper panel is PM. Direction-specific activity in this panel is absent in neck (NF recruited prior to NE) and trunk (RA recruited without TE or LE). Both examples at 6 months show direction-specific postural adjustments: direction-specific activity is present at the level of neck an trunk

The direction-specific trials were characterised by variation in which direction-specific muscles were recruited, in the timing and the amplitudes of the phasic bursts. The 4-months-olds also varied in preference pattern (Fig. 3). But at 6 months - in both conditions-a preference for the complete pattern, i.e. the pattern during which NE, TE and LE were activated in concert, emerged (Fig. 3; age effect, Wilcoxon supine: $p=0.02$ sitting: $p=0.03$ ). This finding differs from the Van der Fits's data (1999a, b), which

Table 2 Median (range) of percentage direction specific trials

\begin{tabular}{ll}
\hline & $\begin{array}{l}\text { Direction } \\
\text { specificity }(\%)\end{array}$ \\
\hline 4 month supine & $60(33-100)$ \\
4 month sitting & $50(13-100)$ \\
6 month supine & $50(29-100)$ \\
6 month sitting & $50(25-73)$ \\
\hline
\end{tabular}



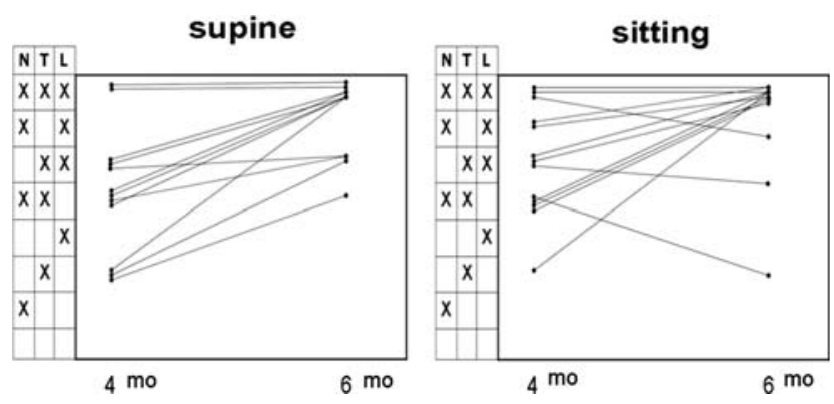

Fig. 3 Individual developmental trajectories of the preference patterns between four and 6 months. Each line represents the development of one infant. $N=$ neck extensor, $T=$ thoracal extensor, $L=$ lumbar extensor. $\boldsymbol{X}$ indicates participation of a direction-specific muscle in a pattern. Three $X$ 's represents the complete pattern. Age-effect of the preference pattern, Wilcoxon, supine: $p=0.02$, sitting: $p=0.03$

indicated that selection of the complete pattern emerged after the age of 6 months.

The latencies to recruitment of the direction-specific muscles were not affected by age or condition (Table 3 ). Nevertheless, a developmental trend in recruitment order could be distinguished. At 4 months about $20-30 \%$ of the trials showed top-down recruitment. At 6 months, a similar rate of top-down recruitment was found in supine position. But in the sitting condition it had increased upto $65 \%$ (Fig. 4; Wilcoxon: $p=0.004$ ).

\section{Kinematics of reaching}

Typical examples of the kinematics of reaching are presented in Fig. 5. At 4 months of age the infants showed a variable number of MU in supine and sitting position. The number varied between 3 and $6 \mathrm{MU}$ and was independent of position. In supine position, the number of MU did not change with increasing age, but in sitting it did: at 6 months sitting infants used less MU than at 4 months (Mann-Whitney $U: p=0.01$; Table 4).

Reaching movements at 4 and 6 months lasted for about $0.5 \mathrm{~s}$ (median values $0.50-0.67 \mathrm{~s}$; Table 4 ). In supine movement duration did not change with age, but in sitting it decreased significantly from $0.67 \mathrm{~s}$ at 4 months to $0.54 \mathrm{~s}$ at 6 months (Mann-Whitney $U: p=0.047$; Table 4).
Similar developmental changes were found in the relative duration of the transport unit. In supine position the transport unit covered about $30 \%$ of reaching duration at both ages, but in sitting the relative duration changed from $28 \%$ at 4 months to $37 \%$ at 6 months (Mann-Whitney $U$ : $p=0.01$; Table 4).

\section{Postural activity affects reaching}

In order to see whether the presence of direction-specific postural activity affected the success of reaching, we classified infants as 'direction-specific' when at least 50\% of trials in a specific condition showed direction-specific postural activity.

In supine position, the presence of direction-specificity tended to be related to success of reaching at 4 months: reaching ended in successful touching or grasping in $76 \%$ of the trials of infants with direction-specific postural activity and in $40 \%$ (median values) of the trials of infants who lacked sufficient direction-specific activity. The difference did however not reach statistical significance. At 6 months the success of reaching in supine position was not affected by the presence of direction-specific activity ('directionspecific' infants: 93\% of reaches successful, 'non-directionspecific' infants: 100\%). However, in sitting the presence of direction specificity was related to success of reaching.

At 4 months reaching during sitting ended in successful touching or grasping of the toy in $46 \%$ of the trials of infants with direction-specific postural activity and in $0 \%$ (median values) of the trials of infants who lacked sufficient direction-specific activity (Mann-Whitney $U: p=0.004$ ). At 6 months reaching during sitting was successful in $81 \%$ of the trials of 'direction-specific' infants and in $36 \%$ of the trials of infants with limited direction-specific activity (Mann-Whitney $U: p=0.046$ ).

At 4 months of age, the presence or absence of consistent direction-specific postural activity did not affect the kinematical characteristics of the successful reaches. But at 6 months reaches in supine position, which were accompanied by direction-specific activity had less MU and a relatively longer duration of the transport MU than reaches without direction specific activity (both effects: MannWhitney: $p=0.01$ ). The effect of direction-specificity on

Table 3 Median (range) latencies to recruitment of the direction-specific dorsal muscles (ms)

\begin{tabular}{llll}
\hline & Neck extensor & Thoracal extensor & Lumbar extensor \\
\hline 4 month supine & $140(-130$ to 1046$)$ & $256(-152$ to 1210$)$ & $229(-78$ to 1104$)$ \\
4 month sitting & $504(-198$ to 1584$)$ & $241(-156$ to 1534$)$ & $462(-20$ to 816$)$ \\
6 month supine & $464(-178$ to 1920$)$ & $329(-200$ to 1814$)$ & $404(-130$ to 1844$)$ \\
6 month sitting & $295(-180$ to 1776$)$ & $418(-196$ to 1724$)$ & $338(-170$ to 1922$)$ \\
\hline
\end{tabular}

Note that latencies in young infants can be rather long 
Fig. 4 Frequency of top-down recruitment at 4 and 6 months in supine and sitting positions. Bold horizontal lines indicate median values, the boxes represent interquartile ranges and the vertical lines total ranges.

$* *$ Wilcoxon $p<0.01$
Fig. 5 Typical examples of the velocity profile of the wrist marker during a reaching movement in supine and sitting position of an infant at 4 and 6 months of age. Dotted vertical lines denote the onset of the reaching movement. Blocks indicate the detected Movement Units (MU). The arrows indicate the first MU, which is used to calculate the relative duration of the first MU. The first MU is in general the MU with the longest duration, in particular in older subjects. Note the differences with older children (2-11 years of age), who show median values of 1 MU per reaching movement and have reaching movements with a total duration of about $1 \mathrm{~s}$. In older children $98 \%$ of the reaching movement is covered by the first MU (Van der Heide et al. 2005)
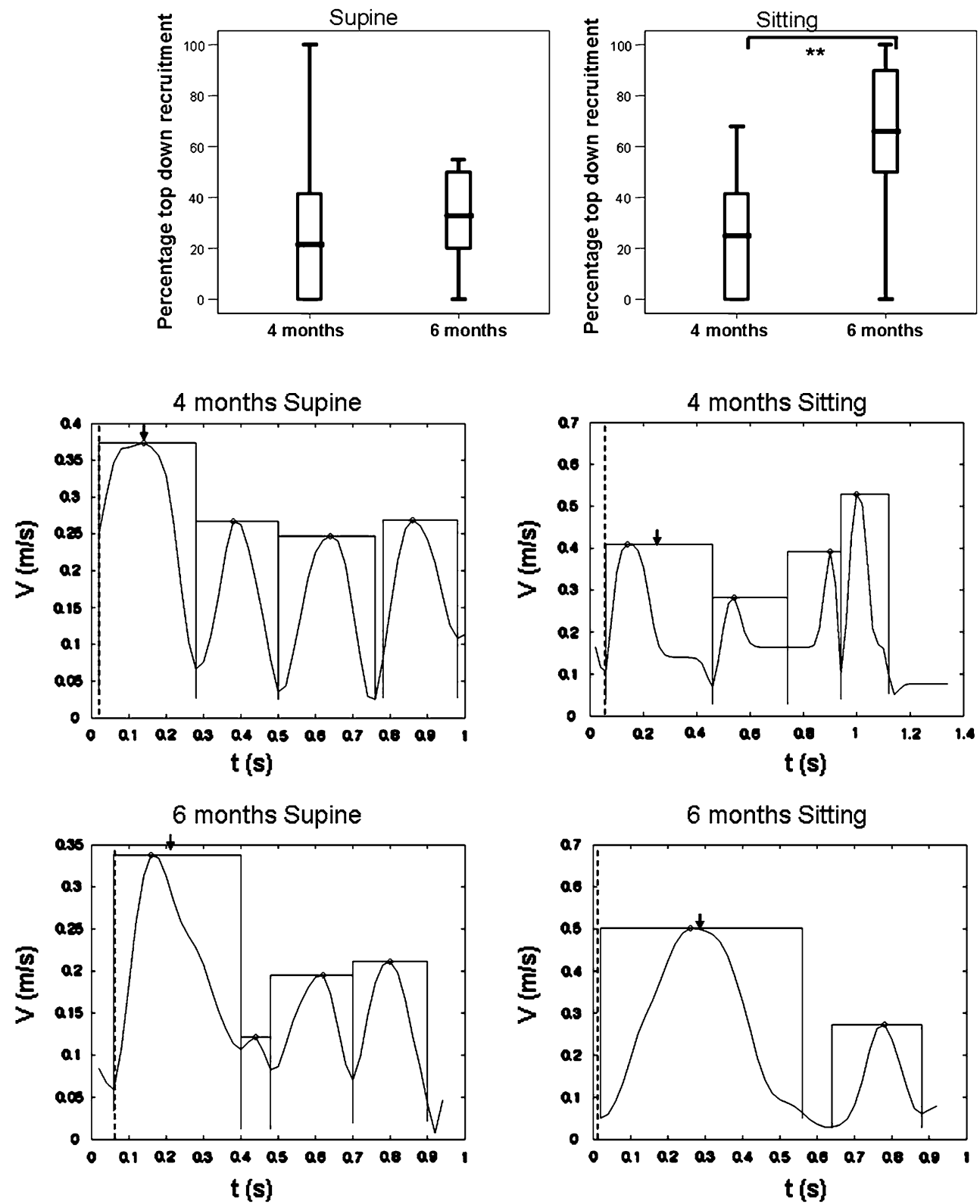

the relative duration of the transport MU was also present in sitting position (Mann-Whitney: $p=0.003$ ). Directionspecificity was not related to the duration of reaching.

The relative duration of the transport unit-and not the number of MU or the total duration of the reaching movement-was also related to other postural characteristics. At 6 months, the relative duration of the transport MU of reaching movements which were accompanied by the complete postural pattern in which NE, TE and LE were activated in concert, was significantly longer than that in reaching movements accompanied by other directionspecific postural patterns (Mann-Whitney $U$ supine: $p=0.03$; sitting: $p=0.02$; Table 5). In addition, the relative duration of the transport MU was longer during reaching movements accompanied by direction-specific activity with top-down recruitment than during reaches without top-down recruitment. The effect was present at both ages and in both conditions (Fig. 6).

\section{Discussion}

The present study demonstrated that about $50 \%$ of reaching movements in lying and sitting infants aged 4 and 6 months are accompanied by direction-specific postural adjustments. At 4 months variation dominates, but at 6 months a preference to recruit muscles in a top-down order (during sitting) and in the configuration of the complete pattern (both conditions) has emerged. Interestingly, the postural characteristics were related to the success and the kinematics of reaching. 
Table 4 Median (range) of kinematical characteristics of reaching movements at different ages and positions

\begin{tabular}{llll}
\hline & Number of MU & Total duration (s) & $\begin{array}{l}\text { Relative duration } \\
\text { MU 1 (\%) }\end{array}$ \\
\hline $\begin{array}{l}\text { 4 months } \\
\text { Supine }\end{array}$ & $4.3(3-6)$ & $0.58(0.44-1.02)$ & $30(14-35)$ \\
Sitting & $3.5(3-5)$ & $0.67(0.32-0.82)$ & $28(18-37)$ \\
6 months & & & \\
Supine & $3.0(2-5.5)$ & $0.50(0.27-0.99)$ & $31(11-48)$ \\
Sitting & $2.5 *(2-3)$ & $0.54 *(0.24-0.58)$ & $37 *(33-50)$ \\
\hline
\end{tabular}

Sitting, differences between 4 and 6 months: Mann-Whitney $U$ $* p<0.05 ; * * p<0.01$

Our findings differ from those of the Van der Fits studies. The differences mainly can be attributed to the more precise definition of direction-specific trials in the present study. The more precise definition resulted in substantially lower rates of direction-specificity. The studies of Hedberg et al. (2004, 2005) indicated that direction-specificitydefined in the same precise manner as in the current study - is virtually always present in young sitting infants when their balance is grossly perturbed by a sudden movement of the support surface. The postural threat during reaching while lying supine or while sitting supported is considerably less. Apparently young infants adopt direction-specific activity only in 50\% of trials in this less dangerous situation. The study of Van der Heide et al. (2003), showed that older children do use consistently directionspecific adjustments while reaching in a stable sitting position. The youngest children whom they had studied were 2 years old. This means that it is currently unclear at which age the consistent recruitment of direction-specificity emerges. The finding that only $50 \%$ of reaching movements of young infants were accompanied by direction-specificity also indicates that direction-specificity is not a prerequisite for the generation of reaching movements.

The more precise definition of the direction-specific trials may also explain why our developmental results differed from the Van der Fits et al. studies. Due to their more liberal definition of direction-specificity they first found a decrease in number of muscles recruited with increasing age. First after the age of 6 months an increase in number of muscles recruited was reported, including the increased selection of the complete pattern. By including only strictly defined direction-specific trials into the developmental analyses, we were able to find developmental trends, which previously remained obscure. In this way we were able to show that within the age period of 4-6 months infants develop the capacity to select 'better' postural patterns, i.e. postural activity which was associated with reaching movements with a better kinematic quality.

At 6 months the infants significantly more often selected the complete pattern in which all dorsal neck and trunk muscles were activated in concert and a postural adjustment with top-down recruitment. The selection of top-down recruitment was only found in the posturally more challenging position of sitting. The developmental sequence from variation to selection fits very well to the ideas of the Neuronal Group Selection Theory (NGST: Edelman 1989; Hadders-Algra 2000). According to NGST motor development is characterised by two phases of variability. During the phase of primary variability motor behaviour is variable, but not geared to external conditions. Next, the phase of secondary variability takes over, during which motor performance can be adapted to specific situations. The transition from primary to secondary variability occurs at function-specific ages. The current data indicate that with respect to the development of postural adjustments during reaching the transition occurs between 4 and 6 months.

Our study underscores the notion of the importance of postural control for the success and quality of reaching. The data indicated that-although direction-specificity turned out not to be a prerequisite for being able to reach-the presence of direction-specific activity in sitting resulted in a higher proportion of reaches which ended successfully in touching or grasping of the object. The effect was not present in supine position, which probably is due to the stable nature of this position. The stability and ease of the supine position compared to that of the sitting position is illustrated by the higher rate of success of reaching in the former situation.

Table 5 Median (range) of relative duration of 1st MU in relation to the organization of the postural adjustment

\begin{tabular}{lllll}
\hline & \multicolumn{2}{l}{ Relative duration of 1st MU (\%) } & & Top down \\
\cline { 2 - 5 } & $\begin{array}{l}\text { Complete pattern } \\
\text { (NE+TE+LE) }\end{array}$ & Other pattern & $\begin{array}{l}\text { No top down } \\
\text { recruitment order }\end{array}$ & $5(3-12)$ \\
\hline 4 month supine & $21(13-30)$ & $12(5-15)$ & $16 *(15-20)$ & $9(4-11)$ \\
4 month sitting & $38(24-75)$ & $11(4-100)$ & $29 *(20-44)$ & $9(6-13)$ \\
6 month supine & $39 *(11-100)$ & $10(6-12)$ & $38 *(12-100)$ & $11(8-12)$ \\
6 month sitting & $29 *(24-56)$ & $17(12-19)$ & $23 *(12-66)$ & \\
\hline
\end{tabular}

Effect of specific postural characteristic: Mann-Whitney $U^{*} p \leq 0.05$ 


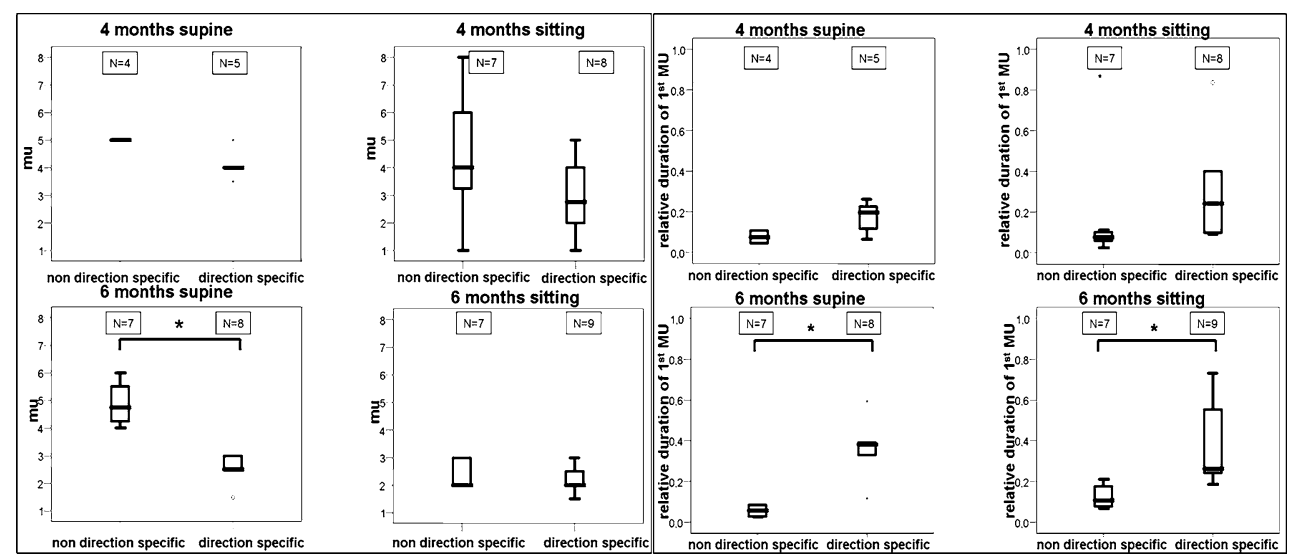

Fig. 6 Left half: Number of MU during reaching in trials with direction-specific postural activity and in trials in which direction-specific activity was not present. Right half: Relative duration of the transport MU (the first MU) during reaching in trials with direction-specific postural activity and in trials in which direction-specific activity was not

At the age of 4 months the organization of postural adjustments only had a minor effect on the kinematical quality of reaching movements. At this age only the presence of top-down recruitment was related to a longer relative duration of the transport MU. A finding which might indicate that also in early infancy head stabilization in space is a major goal in postural control (Pozzo et al. 1990). It is noteworthy that elongation of the transport MU correlated in particular to improved postural control, not only at 4 months but also and even more so at 6 months. As the transport MU is the part of the reaching movement which is largely determined by feedforward programming (Von Hofsten et al. 1998), the finding suggests that between 4 and 6 months feedforward processes become increasingly important in the control of reaching and its associated postural adjustments (cf. Massion 1998).

\section{Concluding remarks}

The present study showed that in early infancy postural adjustments during reaching are not consistently accompanied by direction-specificity and that the presence of direction-specific postural activity is not a prerequisite for successful reaching. Postural development proceeded from variable activity at 4 months to a preference for the in-concert activation of the direction-specific neck- and trunk muscles and-in sitting position only-a preference for top-down recruitment. Our study was the first one to demonstrate that markers of better postural control in young infants such as direction-specificity, selection of the complete pattern and the presence of top-down recruitment were related to more success in reaching and a better kinematical quality of reaching movements. present. The upper panels show data at 4 months, the lower panels at 6 months. In each half-figure, the left panels show data on supine position and right panels the data on sitting position. Bold horizontal lines indicate median values, the boxes represent interquartile ranges and the vertical lines complete ranges. *Mann-Whitney $U, p=0.02$

Acknowledgments We kindly acknowledge Oebo Brouwer, MD, $\mathrm{PhD}$ and Eva Brogren Carlberg, PT, PhD for critical comments on a previous version of this manuscript. The study was supported by the Johanna KinderFonds, Stichting Fonds de Gavere, Cornelia Stichting and the Post-graduate School BCN Groningen.

\section{References}

Amiel-Tison C, Grenier A (1983) The neurological examination of the newborn and infant. Masson, New York

Edelman GM (1989) Neural Darwinism. The theory of neuronal group selection. Oxford University Press, Oxford

Fallang B, Saugstad OD, Hadders-Algra M (2000) Goal directed reaching and postural control in supine position in healthy infants. Behav Brain Res 115:9-18

Forssberg H, Hirschfeld H (1994) Postural adjustments in sitting humans following external perturbations: muscle activity and kinematics. Exp Brain Res 97:515-527

Hadders-Algra M (2000) The neuronal group selection theory: a framework to explain variation in normal motor development. Dev Med Child Neurol 42:566-572

Hadders-Algra M (2005) Development of postural control during the first 18 months of life. Neural Plast 12:99-108

Hedberg A, Forssberg H, Hadders-Algra M (2004) Early development of postural adjustments in sitting position: evidence for the innate origin of direction specificity. Exp Brain Res 157:10-17

Hedberg Å, Brogren Carlberg E, Forssberg H, Hadders-Algra M (2005) Development of postural adjustments in sitting position during the first half year of life. Dev Med Child Neurol 47:312320

Hopkins B, Rönnqvist L (2002) Facilitating postural control: effects on the reaching behavior of 6-month-old infants. Dev Psychobiol 40:168-182

Massion J (1998) Postural control systems in developmental perspective. Neurosci Biobehav Rev 22:465-472

Out L, Savelsbergh GJP, van Soest AJ, Hopkins B (1997) Influence of mechanical factors on movement units in infant reaching. Hum Mov Sci 16:733-748

Piper MC, Darrah J (1994) Motor assessment of the developing infant. WB Saunders, Philadelphia 
Pozzo T, Berthoz A, Lefort L (1990) Head stabilisation during various locomotor tasks in humans. I. Normal subjects. Exp Brain Res 82:97-106

Prechtl HFR (1977) The neurological examination of the full-term newborn infant. In: Clin dev med no. 63, 2nd edn. Heinemann Medical Books, London

Rochat P (1992) Self-sitting and reaching in 5-8-month-old infants: the impact of posture and its development on early eye-hand coordination. J Mot Behav 24:210-220

Savelsbergh GJP, van der Kamp J (1994) The effect of body orientation to gravity on early infant reaching. J Exp Child Psychol 58:510 528

Thelen E, Spencer JP (1998) Postural control during reaching in young infants: a dynamic systems approach. Neurosci Biobehav Rev 22:507-514

Thelen E, Corbetta D, Kamm K, Schneide K, Zernicke RF (1993) The transition to reaching: mapping intention and intrinsic dynamics. Child Dev 64:1058-1098

Touwen B (1976) Neurological development in infancy. In: Clin dev med no. 58. Heinemann Medical Publications, London

Trevarthen C (1984) How control of movements develops. In: Whiting HTA (ed) Human motor actions. Bernstein reassessed. Elsevier, Amsterdam, pp 223-261
Van der Fits IBM, Hadders-Algra M (1998) The development of postural response patterns during reaching in healthy infants. Neurosci Biobehav Rev 22:521-526

Van der Fits IBM, Klip AWJ, van Eykern LA, Hadders-Algra M (1999a) Postural adjustments during spontaneous and goal directed arm movement in the first half year of life. Behav Brain Res 106:75-90

Van der Fits IBM, Otten E, Klip AWJ, Van Eykern LA, Hadders-Algra M (1999b) The development of postural adjustments during reaching in 6-18-month-old infants: evidence for two transitions. Exp Brain Res 126:517-528

Van der Heide JC, Otten B, Van Eykern LA, Hadders-Algra M (2003) Development of postural adjustments during reaching in sitting children. Exp Brain Res 151:32-45

Van der Heide JC, Fock JM, Otten E, Stremmelaar E, Hadders-Algra M (2005) Kinematic characteristics of reaching movements in preterm children with cerebral palsy. Pediatr Res 57:883-889

Von Hofsten C (1982) Eye-hand coordination in the newborn. Dev Psychol 18:450-461

Von Hofsten C (1991). Structuring of early reaching movements: a longitudinal study. J Mot Behav 23:280-292

Von Hofsten C, Vishton P, Spelke ES, Feng Q, Rosander K (1998) Predictive action in infancy: tracking and reaching for moving objects. Cognition 67:255-285 